\title{
sciendo
}

\section{Lower-Body Power Relationships to Linear Speed, Change-of-Direction Speed, and High-Intensity Running Performance in DI Collegiate Women's Basketball Players}

\author{
by \\ Daveena S. Banda', Maria M. Beitzel' ${ }^{1}$ Joseph D. Kammerer ${ }^{1,2}$, Isaac Salazar², \\ Robert G. Lockie ${ }^{1}$
}

\begin{abstract}
Basketball players need to sprint and change direction, and lower-body power (often measured by jump tests) should contribute. How different jumps relate to linear and change-of-direction (COD) speed, and high-intensity running has not been analyzed in Division I (DI) collegiate women's basketballers. Twelve players completed the vertical jump (VJ), two-step approach jump (AppJ), and standing broad jump (SBJ). Average (AvgP) and peak power (PeakP), and PeakP: body mass (P:BM) were derived from VJ height; relative SBJ was derived from SBJ distance. Players also completed: $10 \mathrm{~m}$ and $3 / 4$ court sprints (linear speed), the pro-agility shuttle (COD speed), and the Yo-Yo Intermittent Recovery Test Level 1 (YYIRT1; high-intensity running). Pearson's correlations $(p<0.05)$ calculated relationships between the jump and running tests. The AppJ correlated to the $3 / 4$ court sprint and pro-agility shuttle $(r=$ -.663 to -.805). AvgP and PeakP correlated to the $10 \mathrm{~m}$ sprint, $3 / 4$ court sprint, and pro-agility shuttle $(r=.589-.766)$. $P: B M$ and relative SBJ correlated with all running tests (linear and COD speed $r=-.620$ to -.805 ; YYIRT1 $r=.622-$ .803). The AppJ stresses the stretch-shortening capacities of the legs, and this quality is important for faster linear and COD speed. AvgP and PeakP are influenced by body mass; while larger athletes produce greater power, they also may display slower $10 \mathrm{~m}$ sprint and pro-agility shuttle times, and lesser YYIRT1 performance. Strength coaches should ensure players can generate high relative power (i.e. P:BM, relative SBJ) for faster linear and COD speed, and highintensity running.
\end{abstract}

Key words: agility, female athletes, jump tests, quickness, relative power, sprinting.

\section{Introduction}

Collegiate basketball players are reliant on the ability to sprint and change direction at high speeds, which are necessary qualities to play the game effectively (Spiteri et al., 2015). The better an athlete is at these qualities, the more likely they are to be considered successful at their sport. In addition to these attributes, highintensity running is also an important characteristic for basketball players to possess (Castagna et al., 2008). In fact, throughout the duration of a game, basketball athletes can complete 40-60 short sprints, more than 40 jumps, and approximately 100 high-intensity basketballspecific actions that involve direction changes (Ben Abdelkrim et al., 2007). This emphasizes the importance of these physical attributes. Faster execution of sprinting and change-of-direction (COD) movements has also been associated with more playing time in collegiate basketball (Hoffman et al., 1996). Several studies suggest a relationship between lower-body power and linear speed, COD speed, and high-intensity running (Cronin and Hansen, 2005; Lockie et al., 2016; McFarland et al., 2016; Mikołajec et al., 2012; Padulo et al., 2015). Lower-body power can be

1 - Center for Sport Performance, Department of Kinesiology, California State University, Fullerton, Fullerton, CA, USA.

2 - Strength and Conditioning, Department of Intercollegiate Athletics, California State University, Fullerton, CA, USA. 
measured in different ways. Jump tests are commonly used to indirectly measure this quality, as power can be calculated from these measurements (Harman et al., 1991).

Performance of jump tests has previously been linked to sprinting performance over a variety of distances and directions in athletic populations (Lockie et al., 2016; McFarland et al., 2016; Padulo et al., 2015). Specifically, several studies have reported significant relationships between better performance in jump tests (e.g. vertical jump [VJ], bilateral and unilateral standing broad jump [SBJ], squat jumps) and faster linear speed (Lockie et al., 2014, 2016; Shalfawi et al., 2011; Wisløff et al., 2004). This implies that better performance in certain jump tests may actually translate to better sprint performance in game-like settings. For example, Wisløff et al. (2004) reported that VJ performance correlated with both 10 meter $(\mathrm{m})$ and $30 \mathrm{~m}$ sprint time $(r=0.60-0.72, p<0.01)$. These results illustrate the significance of lower-body power and its relation to linear sprint performance. However, there is limited research regarding this relationship in Division I (DI) collegiate female basketball players.

Many team sports require the ability to change direction while sprinting. In previous literature, successful COD ability appears to require prerequisites such as lower-body strength and power (Chaouachi et al., 2012; McFarland et al., 2016; Young et al., 1995). Furthermore, jump tests used as a measure of power, such as the VJ and squat jump, have been found to relate to COD speed. For example, McFarland et al. (2016) found significant relationships between the $\mathrm{VJ}$ and squat jump with pro-agility shuttle and T-test times $(r=$ -0.50 to $-0.79, p<0.05)$ in collegiate women soccer players. Notably, this included VJ height, in addition to peak power derived from VJ height relative to body mass (McFarland et al., 2016). Possessing appropriate levels of these qualities should allow an athlete to seamlessly transition from one direction change to the next, which should be desirable during a basketball game. Therefore, jump performance should relate to COD speed in DI collegiate women basketball players, although this is still to be confirmed.

Another important quality shown to benefit performance in team sports is the ability to repeatedly sprint with maximal effort (Gabbett et al., 2013; Maggioni et al., in press; Padulo et al., 2015). Basketball in particular is a sport that requires short bursts of repeated high-intensity actions (Attene et al., 2015; Ben Abdelkrim et al., 2007; Padulo et al., 2015). A test that is often used to assess maximal aerobic capacity $\left(\mathrm{VO}_{2 \max }\right)$ and high-intensity running performance in athletes is the Yo-Yo Intermittent Recovery Test Level 1 (YYIRT1) (Bangsbo et al., 2008; Castagna et al., 2008). YYIRT1 performance and $\mathrm{VO}_{2 \max }$ values in basketball players were significantly related, demonstrating the YYIRT1 as a valid test to measure aerobic-fitness in basketball players (Castagna et al., 2008). Further to this, it could be expected that these short-burst running actions in collegiate women's basketball players could be augmented by lower-body power, as relationships between repeated-sprint ability and the VJ and SBJ have been shown in collegiate men's soccer players (Lockie et al., 2019). Nonetheless, the relationship between jump tests and highintensity running measured by the YYIRT1 remains unclear, and this is particularly true in DI collegiate women basketball players.

Therefore, the purpose of this study was to determine relationships between lower-body power measured via jump tests with linear speed, COD speed, and high-intensity running performance in DI collegiate women's basketball players. A correlation analysis of a female DI collegiate basketball team was conducted using sport-specific field tests, including: VJ, approach vertical jump (AppJ) and SBJ; $10 \mathrm{~m}$ and $3 / 4$ court linear sprint tests; the pro-agility shuttle; and the YYIRT1. It was hypothesized that the jump tests would correlate with performance in the running tests examined; specifically, that the players with higher power output, measured in both absolute and relative terms, would perform better in the linear, COD, and high-intensity running tests.

\section{Methods}

\section{Participants}

A retrospective analysis of existing data was conducted on one women's DI collegiate basketball team, which encompassed 12 players (body height $=1.75 \pm 0.09 \mathrm{~m}$; body mass $=73.37 \pm$ $17.30 \mathrm{~kg}$ ). Age for the players was not provided in the data set, but the team was typical of collegiate female basketball players (Payne et al., 1997; Pfile et al., 2016). All players were required to be 
actively competing and training with the team, and were injury-free at the time of testing. The data used in this study arose as a condition of monitoring conducted by the team's coaching staff. As a result, the California State University, Fullerton institutional review board approved the use of pre-existing data (HSR-18-19-121). Nonetheless, the study still conformed to the recommendations of the Declaration of Helsinki. Each player had also completed the universitymandated physical examination, and read and signed the university consent and medical forms for participation in collegiate athletics.

Procedures

The team's coaching staff tested all players. This testing protocol was conducted before and after the season to evaluate whether the strength and conditioning program was effective. Firstly, players had their body height and mass recorded. Body height was measured barefoot using a portable stadiometer (Detecto, Webb City, MO, USA), while mass was recorded by electronic digital scales (Ohaus, Parsippany, NJ, USA). Each of the player's body mass was given in pounds and converted to kilograms. All athletes were familiar with the tests completed in this study, as they were performed as part of standard physical monitoring practices by the team's coaching staff. These assessments were administered during the pre-season in the summer, in-season in the first week of October, and post-season in March. The data analyzed in this study were taken from the pre-season period.

All three jump tests were conducted in one session, and the sprint tests were conducted in another session. Before initiating each of the three jump tests, a dynamic warm-up was performed in eight movements: lunge and twist, inchworm and frog, up dog down dog, scorpion kicks, knee hugs, overhead squat, pigeon plus twist, and band shoulder rotations. Additionally, there was a jump rope warm-up: 100 repetitions, jump warm-up, and then a jump and stick completed five times. The Brower Vertical Jump system (BVJ; Brower Timing System, UT, USA) was used to measure each of the athlete's full reach. This was done by having each athlete stand alongside the BVJ and extend with their dominant arm. The first assessments were of lower-body power tests including the VJ, SBJ, and AppJ tests. Each of these indirect assessments of bilateral lower-body power was measured in centimeters $(\mathrm{cm})$. Three trials for each jump test were completed, with the best trial analyzed. The running tests were performed on the basketball court where the players regularly trained. Players completed the $10 \mathrm{~m}$ and $3 / 4$ court sprint (measured in seconds [s]) to measure linear speed, the proagility shuttle to measure COD speed (measured in s), and the YYIRT1, with the number of shuttles completed providing a measure of aerobic fitness and high-intensity running capacity (Bangsbo et al., 2008; Castagna et al., 2008). Depending on coach preference with a particular athlete, 2-3 attempts were provided for the running speed tests, except for the YYIRT1, where only one attempt was completed.

\section{Vertical Jump (VJ)}

The VJ was used to indirectly measure lower-body power in the vertical plane. This test abided by previous guidelines outlined in the literature (Lockie et al., 2012). The BVJ (Brower Timing System, UT, USA) was used to measure the jumps. Athletes initially stood with their dominant side toward the BVJ, and while facing forwards and keeping their heels on the ground, reached upward as high as possible. This allowed the coaching staff to record their highest reach in order to calculate displacement once the athletes completed their jumps. Further instructions included jumping as explosively as possible and extending their dominant hand (along the BVJ) as high as they could. Each athlete had 2-3 attempts to jump as high as they could. The highest jump was recorded and converted to $\mathrm{cm}$. Power was calculated from the VJ through the formulas: Average power $(\operatorname{AvgP}$; measured in watts $[\mathrm{w}])=$ $21.2 \times$ jump height $(\mathrm{cm})+23.0 \times$ body mass $(\mathrm{kg})-$ 1391; Peak power (PeakP; measured in watts [w]) $=61.9 \times$ jump height $(\mathrm{cm})+36.0 \times$ body mass $(\mathrm{kg})$ + 1822 (Harman et al., 1991). PeakP was also used to calculate a power: body mass ratio (P:BM) via the equation: $P: B M=$ PeakP $\cdot B M^{-1}$ (McFarland et al., 2016).

\section{Two-step Approach Jump (AppJ)}

The AppJ was conducted using a previously established approach (Pehar et al., 2017). The BVJ was again used to record jump height. The athletes used an individually determined running approach (maximum $5 \mathrm{~m}$ distance from the start to take-off, which would encompass two steps) and performed a bounce 
jump with an arm swing. This task was followed by a quick upward vertical jump, accompanied with one-arm maximal reach height. The athletes were instructed to perform the jumping procedure in the way that they found most convenient. This particular jump test was highly encouraged to mimic the athlete's personal technique as they would use in a game or practice situation (Pehar et al., 2017).

\section{Standing Broad Jump (SBJ)}

The bilateral SBJ was used to measure lower-body power in the horizontal plane. This test was completed following similar test structures used in previous studies (Lockie et al., 2012, 2018b). In the SBJ, athletes were directed to jump as far as they could from a designated starting point. This required both feet to be behind the starting point to initiate the test. No restrictions were given for the range of the countermovement or the degree of arm swing used. In order for the jump distance to be recorded, they needed to land with both feet grounded; if not, the trial was reattempted. The foot closest to the starting point was the noted to calculate their horizontal displacement with a standard measuring tape (Lockie et al., 2012). SBJ distance was made relative to body mass via the formula: relative standing broad jump $=$ jump distance.body mass $^{-1}$ (Lockie et al., 2012).

\section{$10 \mathrm{~m}$ Sprint}

The $10 \mathrm{~m}$ sprint was used to measure linear speed. The protocol for this test followed previously established guidelines (Lockie et al., 2018a), and $10 \mathrm{~m}$ sprint time was recorded by a timing lights system (PowerMax TC Gates; Brower Timing System, UT, USA). Timing gates were positioned at 0 and $10 \mathrm{~m}$. Athletes were directed to begin from $50 \mathrm{~cm}$ behind the actual start line in order to initiate the first set of timing gates. Athletes were also instructed to run maximally from the beginning through the last set of timing gates.

\section{$3 / 4$ Court Sprint}

The $3 / 4$ court sprint was used to measure both linear speed and acceleration. Sprint time was recorded by timing gates (PowerMax TC Gates; Brower Timing System, UT, USA). Athletes were familiar with the protocol of the $3 / 4$ court sprint prior to testing. This test involves athletes sprinting maximally for a $22 \mathrm{~m}$ distance, with the gates positioned at 0 and $22 \mathrm{~m}$.

\section{Pro-agility Shuttle}

The test was completed using established methods (Lockie et al., 2012, 2018b), and is shown in Figure 1. Athletes straddled the middle line in a 3-point stance in between the timing gate. As per the timing system set-up, one timing gate (PowerMax TC Gates; Brower Timing System, UT, USA) was used. Once the athlete was stable in their 3-point stance they could begin the test. Timing was initiated by the first movement of the hand. To start the test, the athlete turned and ran $4.57 \mathrm{~m}$ to one side and touched the line with one hand. The athlete then turned and ran $9.14 \mathrm{~m}$ to the other side and touched the other line, before turning and finishing by running back through the start/finish line. Coaches were positioned at either end of the pro-agility shuttle to ensure athletes touched the line. If they did not, the trial was disregarded and reattempted. The timing system started when the athlete left the light beam and stopped recording when athletes returned through the gate for the last time.

\section{YYIRT1}

The YYIRT1 was administered based on previous guidelines (Bangsbo et al., 2008). The YYIRT1 is characterized by 20-m shuttle runs with $10 \mathrm{~s}$ of recovery between each run. The YYIRT1 has four running bouts at 10-13 kilometers per hour $\left(\mathrm{km} \cdot \mathrm{hr}^{-1}\right)$, and another seven runs at 13.5-14 $\mathrm{km} \cdot \mathrm{hr}^{-1}$. Following this, the YYIRT1 continues with stepwise $0.5 \mathrm{~km} \cdot \mathrm{hr}^{-1}$ speed increments after every eight running bouts until exhaustion (Lockie et al., 2018b). The test was considered to be over in two instances: one, when the player failed twice to reach the finishing line in time; or two, when the player was physically unable to complete another shuttle at the designed speed. The total number of shuttles completed was considered the final test score.

\section{Statistical Analysis}

All statistical analyses were computed using the Statistics Package for Social Sciences (Version 25.0; IBM Corporation, New York, USA). Descriptive statistics (mean \pm standard deviation [SD]) were calculated for each variable. Pearson's two-tailed correlation analysis determined relationships between the jump tests (VJ, SBJ, and AppJ) with the running performance tests $(10 \mathrm{~m}$ sprint, $3 / 4$ sprint, pro-agility shuttle, and YYIRT1). An alpha level of $p<0.05$ was required for significance. Correlation strength was defined as: 
$r$ between 0 to 0.3 , or 0 to -0.3 , was considered small; 0.31 to 0.49 , or -0.31 to -0.49 , moderate; 0.5 to 0.69 , or -0.5 to -0.69 , large; 0.7 to 0.89 , or -0.7 to 0.89 , very large; and 0.9 to 1 , or -0.9 to -1 , near perfect for relationship prediction (Hopkins, 2013).

\section{Results}

Descriptive data are shown in Table 1, while the correlation data are shown in Table 2. AvgP calculated from the VJ displayed a very large positive correlation to the $10 \mathrm{~m}$ sprint, $3 / 4$ court sprint, and pro-agility shuttle. Additionally, PeakP from the VJ was also positively correlated to the $3 / 4$ court sprint and pro-agility shuttle. The relationship among these variables was indicated to be between a large-to-very large magnitude.
AvgP and PeakP from the VJ were found to be negatively correlated with the YYIRT1, with very large relationships. P:BM had significant, negative relationships with the $10 \mathrm{~m}$ sprint (large), 3/4 court sprint (very large), and pro-agility shuttle (large), and a positive relationship with the YYIRT1 (large). The AppJ was negatively correlated to the $3 / 4$ court sprint and the pro-agility shuttle, and the relationship strength was large and very large, respectively. The relative SBJ had significant, negative relationships with the $10 \mathrm{~m}$ sprint (large), $3 / 4$ court sprint (very large), and pro-agility shuttle (large), and a positive relationship with the YYIRT1 (very large). There were no significant correlations between VJ height and SBJ distance with the high-intensity running tests.

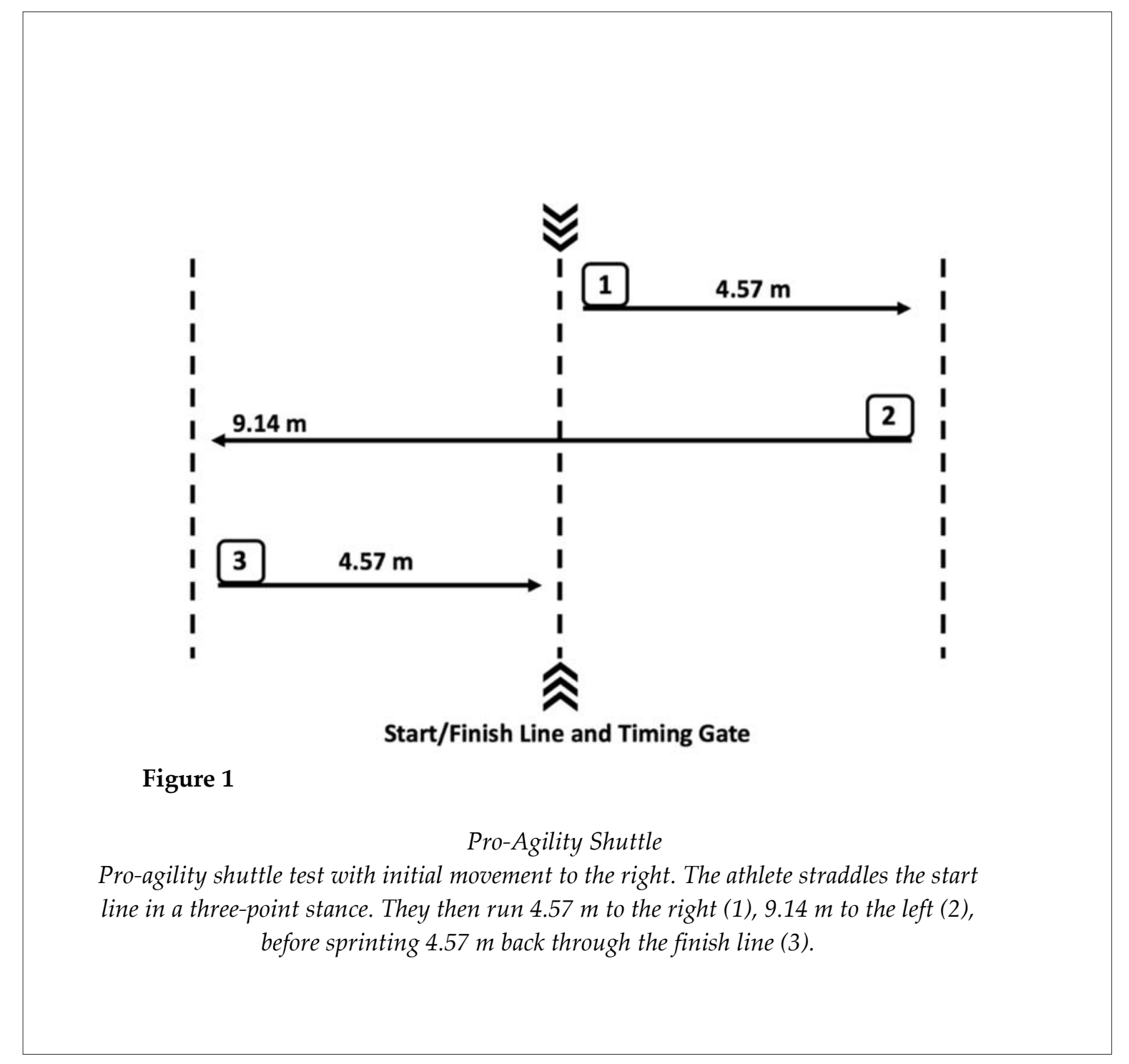


Table 1.

Descriptive data for DI collegiate women's basketball $(N=12)$ players in the: vertical jump, average and peak power derived from VJ height, peak power:body mass ratio, two-step approach jump, standing broad jump distance and relative standing broad jump,

$10 \mathrm{~m}$ sprint, 3/4 court sprint, pro-agility shuttle, and the Yo-Yo Intermittent Recovery

Test Level 1.

\begin{tabular}{lc}
\hline & Mean \pm SD \\
\hline Vertical Jump (cm) & $43.8 \pm 6.87$ \\
Average Power (watts) & $1224.67 \pm 367.89$ \\
Peak Power (watts) & $7179.30 \pm 605.91$ \\
Peak Power: Body Mass Ratio & $100.80 \pm 15.10$ \\
Approach Jump (cm) & $62.01 \pm 7.13$ \\
Standing Broad Jump (m) & $1.99 \pm .146$ \\
Relative Standing Broad Jump $\left(\mathrm{m} \cdot \mathrm{kg}^{-1}\right)$ & $0.029 \pm 0.007$ \\
10 m Sprint (s) & $1.93 \pm .102$ \\
$3 / 4$ Court Sprint (s) & $3.59 \pm .202$ \\
Pro-Agility Shuttle (s) & $4.72 \pm .298$ \\
Yo-Yo Intermittent Recovery Test Level 1 & $27.09 \pm 11.94$ \\
(shuttles) &
\end{tabular}

\section{Table 2}

Pearson's correlations between different measures of lower-body power with the linear and COD speed tests, and the YYIRT1. ${ }^{*}$ Significant $(p<0.05)$ relationship between the two variables.

\begin{tabular}{|c|c|c|c|c|c|c|c|c|}
\hline & & VJ & AvgP & PeakP & $\mathrm{P}: \mathrm{BM}$ & AppJ & SBJ & Rel SBJ \\
\hline \multirow{2}{*}{$10 \mathrm{~m}$ sprint } & $r$ & -.242 & $.658^{*}$ & .547 & $-.620^{*}$ & -.417 & -.289 & $-.628^{*}$ \\
\hline & $p$ & .448 & .020 & .066 & .032 & .178 & .362 & .029 \\
\hline \multirow{2}{*}{ 3/4 Sprint } & $r$ & -.371 & $.766^{*}$ & $.607^{*}$ & $-.758^{*}$ & $-.663^{*}$ & -.478 & $-.758^{*}$ \\
\hline & $p$ & .235 & .004 & .036 & .006 & .019 & .116 & .004 \\
\hline \multirow{2}{*}{$\begin{array}{l}\text { Pro-Agility } \\
\text { Shuttle }\end{array}$} & $r$ & -.365 & $.745^{*}$ & $.589^{*}$ & $-.666^{*}$ & $-.805^{*}$ & -.268 & $-.620^{*}$ \\
\hline & $p$ & .243 & .005 & .044 & .018 & .002 & .400 & .031 \\
\hline \multirow{2}{*}{ YYIRT1 } & $r$ & .020 & -.810 & $-.768^{*}$ & $.622^{*}$ & .278 & .519 & $.803^{*}$ \\
\hline & $p$ & .952 & .003 & .006 & .041 & .408 & .102 & .003 \\
\hline
\end{tabular}




\section{Discussion}

This study observed the relationship between lower-body power measured via jump tests (VJ, AppJ, and SBJ) and how they related to performance in high-intensity running tests $(10 \mathrm{~m}$ sprint, $3 / 4$ court sprint, pro-agility shuttle, and YYIRT1) in DI collegiate women's basketball players. A better AppJ related to a faster 3/4 court sprint and the pro-agility shuttle, which was expected. Interestingly, the results indicated that greater AvgP and PeakP related to a slower $10 \mathrm{~m}$ sprint, $3 / 4$ court sprint, and pro-agility shuttle performance, a fewer distance covered in the YYIRT1. This could be because larger players tended to generate more power, but could not move as effectively in the running tests. The importance of generating power relative to body mass was reinforced by the findings that indicated a greater P:BM and relative SBJ were related to faster linear and COD speed, and more shuttles performed in the YYIRT1. This study provides useful information that could improve the understanding of collegiate women's basketball performance and contribute to new training ideas.

It has been previously shown that performance in certain jump tests have a positive relationship to faster linear (Lockie et al., 2014, 2016) and COD (Lockie et al., 2016; McFarland et al., 2016) speeds in athletic populations. In this study, the results of the AppJ indicated similar relationships as a higher score in this particular jump related to a faster $3 / 4$ court sprint and proagility shuttle in DI collegiate women's basketball players. The AppJ is a type of vertical jump that utilizes the stretch-shortening cycle (SSC) capacities of the legs, and this is an important quality for faster linear and COD speed (Hennessy and Kilty, 2001). In line with these findings for the importance of the SSC, the current results suggested it related to short distance speed ( $3 / 4$ court sprint) and agility (pro-agility shuttle). Both of these capacities have been previously acknowledged as potential indicators of superior collegiate basketball performance (Hoffman et al., 1996). Accordingly, these data suggest collegiate women basketball players should develop their SSC capacities of the legs to enhance their linear and COD speed. A suggested training modality is the incorporation of plyometrics (Lockie et al., 2018a). This type of training can elicit power and explosiveness, both desirable traits for the game of basketball.

Interestingly, even though the $10 \mathrm{~m}$ sprint may also be classified as a short distance speed test, it was not found to be significantly correlated to the AppJ. This may be due in part to the different phases of sprinting, and small yet impactful difference in distance between the two tests. The $10 \mathrm{~m}$ sprint requires athletes to accelerate faster in a shorter period of time, whereas in the $3 / 4$ court sprint, athletes have about 10 additional $\mathrm{m}$ to accelerate and reach close to their top end velocity. Speed over longer distances becomes more dependent on elastic energy provided by the SSC components of the legs (Kraemer et al., 2000), more so than sprint acceleration. Indeed, strength may be a greater contributor to speed over short $10 \mathrm{~m}$ distances than lower-body power (Young et al., 1995). However, further research is needed to confirm this theory.

AvgP and PeakP derived from the VJ are influenced by body mass, and while larger athletes produced higher amounts of power, they also had slower $10 \mathrm{~m}$ sprint and pro-agility shuttle times, and lesser YYIRT1 performance. These findings are supported by previous studies that reported relationships between leg power relative to body mass and sprint performance, irrespective of the distance (Keiner et al., 2014). Although the expression of power is generally encouraged in basketball, power generation relative to body mass is an important consideration.

This was highlighted by the correlation results for the P:BM and relative SBJ. Those players that generated a greater P:BM, and had a higher relative SBJ, tended to be faster in the $10 \mathrm{~m}$ sprint, $3 / 4$ sprint, and pro-agility shuttle, and completed more YYIRT1 shuttles. These data were supported by McFarland et al. (2016) who found that in collegiate soccer players, those players that had a higher P:BM measured in the VJ and squat jump were faster in a $30 \mathrm{~m}$ sprint, pro-agility shuttle, and T-test. The relative SBJ is a more novel relative power measure, which was introduced by Lockie et al. (2012). In an analysis of high school-aged male American football players, Lockie et al. (2012) found that a higher relative SBJ correlated with faster $36.6 \mathrm{~m}$ sprint, pro-agility shuttle, and three-cone drill 
performance. The results from this study reinforced the findings of McFarland et al. (2016) and Lockie et al. (2012), and clearly demonstrated the importance of relative power for DI collegiate women's basketball players. Those players that can generate greater power relative to their body mass will likely be faster in linear and COD speed tasks, and superior in high-intensity running as well.

No significant correlations were found for VJ height or SBJ distance. This was somewhat surprising as previous studies have actually shown a relationship between VJ height and similar sprint tests such as the ones used in this study (Lockie et al., 2016; Shalfawi et al., 2011). In DI collegiate women's basketball players, however, generating power for a raw performance metric is perhaps less important than doing so relative to body mass. In conjunction with the AvgP, PeakP, P:BM, and relative SBJ results from this study, these data highlighted the range of body sizes prevalent in collegiate women's basketball, due to different positional requirements. Larger players tend to play positions such as a power forward and center that demand high lower-body power output, and usually carry more body fat (Delextrat and Cohen, 2009). A higher body mass could be advantageous for athletes that play forward and center in basketball since they are likely to experience more physical contact (Delextrat and Cohen, 2009). Despite this, strength and conditioning coaches should ensure that their larger players, even though they may generate more power to overcome the inertia of their body mass, can still move effectively when sprinting and changing direction, and when they have to complete repeated high-intensity efforts. Indeed, developing high power relative to body mass should be a focus for all collegiate women's basketball players.

There are limitations in this study that should be acknowledged. This study primarily relied on the performance of lower-body power, independent of strength. In previous literature, strength has been shown to contribute to attributes such as sprinting and power production (Keiner et al., 2014; Young et al., 1995). However, lower-body strength was not measured, but should be included in future studies on DI collegiate women's basketball players. Research should include lower-body strength as measured by tests such as the barbell back squat or hex bar deadlift. In addition, this study only analyzed players from one collegiate basketball team. Future studies should also consider testing multiple teams to increase the sample size of collegiate women's basketball players, and to allow for greater utility of the study results to a larger population of athletes.

In conclusion, the ability to generate high amounts of lower-body power as measured by the AppJ was associated with faster performance in linear ( $3 / 4$ court sprint) and COD (pro-agility shuttle) sprinting. Although Avg and PeakP were shown to be correlated with running tests, it should also be noted that just generating high power may not be a sole predictor of performance in high-intensity running tasks. This could be related to the size of players in collegiate women's basketball teams, as larger players tend to generate greater power to overcome their body mass. Indeed, it may be more important for collegiate women's basketball players to generate high power relative to their body mass. This was shown by data that indicated players with a greater $\mathrm{P}: \mathrm{BM}$ and standing SBJ were superior in the $10 \mathrm{~m}$ sprint, $3 / 4$ court sprint, pro-agility shuttle, and YYIRT1. Strength and conditioning coaches for collegiate women's basketball players should ensure that all players, regardless of size, can generate high power relative to body mass. This should positively influence linear speed, COD speed, and high-intensity running.

\section{Acknowledgements}

This research project received no external financial assistance. None of the authors have any conflict of interest. 


\section{References}

Attene G, Laffaye G, Chaouachi A, Pizzolato F, Migliaccio GM, Padulo J. Repeated sprint ability in young basketball players: one vs. two changes of direction (Part 2). J Sports Sci, 2015; 33: 1553-1563

Bangsbo J, Iaia FM, Krustrup P. The Yo-Yo Intermittent Recovery Test. Sports Med, 2008; 38: 37-51

Ben Abdelkrim N, El Fazaa S, El Ati J. Time-motion analysis and physiological data of elite under-19-yearold basketball players during competition. Br J Sports Med, 2007; 41: 69-75

Castagna C, Impellizzeri FM, Rampinini E, D'Ottavio S, Manzi V. The Yo-Yo intermittent recovery test in basketball players. J Sci Med Sport, 2008; 11: 202-208

Chaouachi A, Manzi V, Chaalali A, Wong del P, Chamari K, Castagna C. Determinants analysis of changeof-direction ability in elite soccer players. J Strength Cond Res, 2012; 26: 2667-2676

Cronin JB, Hansen KT. Strength and power predictors of sports speed. J Strength Cond Res, 2005; 19: 349-357

Delextrat A, Cohen D. Strength, power, speed, and agility of women basketball players according to playing position. J Strength Cond Res, 2009; 23: 1974-1981

Gabbett TJ, Wiig H, Spencer M. Repeated high-intensity running and sprinting in elite women's soccer competition. Int J Sports Physiol Perform, 2013; 8: 130-138

Harman EA, Rosenstein MT, Frykman PN, Rosenstein RM, Kraemer WJ. Estimation of human power output from vertical jump. J Strength Cond Res, 1991; 5: 116-120

Hennessy L, Kilty J. Relationship of the stretch-shortening cycle to sprint performance in trained female athletes. J Strength Cond Res, 2001; 15: 326-331

Hoffman JR, Tenenbaum G, Maresh CM, Kraemer WJ. Relationship between athletic performance tests and playing time in elite college basketball players. J Strength Cond Res, 1996; 10: 67-s,

Hopkins WG. A scale of magnitude for effect statistics, 2002 Available at: www.sportsci.org/resource/stats/index.html; accessed on 06.10.2011

Keiner M, Sander A, Wirth K, Schmidtbleicher D. Long-term strength training effects on change-of-direction sprint performance. J Strength Cond Res, 2014; 28: 223-231

Kraemer WJ, Ratamess NA, Volek JS, Mazzetti SA, Gómez AL. The effect of the Meridian shoe on vertical jump and sprint performances following short-term combined plyometric/sprint and resistance training. J Strength Cond Res, 2000; 14: 228-238

Lockie RG, Callaghan SJ, Berry SP, Cooke ER, Jordan CA, Luczo TM, Jeffriess MD. Relationship between unilateral jumping ability and asymmetry on multidirectional speed in team-sport athletes. $J$ Strength Cond Res, 2014; 28: 3557-3566

Lockie RG, Dawes JJ, Jones MT. Relationships between linear speed and lower-body power with change-ofdirection speed in National Collegiate Athletic Association Divisions I and II women soccer athletes. Sports, 2018a; 6: doi:10.3390/sports6020030

Lockie RG, Jeffriess MD, Schultz AB, Callaghan SJ. Relationship between absolute and relative power with linear and change-of-direction speed in junior American football players from Australia. J Aust Strength Cond, 2012; 20: 4-12

Lockie RG, Moreno MR, Lazar A, Orjalo AJ, Giuliano DV, Risso FG, Davis DL, Crelling JB, Lockwood JR, Jalilvand F. The physical and athletic performance characteristics of Division I collegiate female soccer players by position. J Strength Cond Res, 2018b; 32: 334-343

Lockie RG, Moreno MR, Orjalo AJ, Stage AA, Liu TM, Birmingham-Babauta SA, Hurley JM, Torne IA, Beiley MD, Risso FG, Davis DL, Lazar A, Stokes JJ, Giuliano DV. Repeated-sprint ability in Division I collegiate male soccer players: Positional differences and relationships with performance tests. J Strength Cond Res, 2019; 33: 1362-1370

Lockie RG, Stage AA, Stokes JJ, Orjalo AJ, Davis DL, Giuliano DV, Moreno MR, Risso FG, Lazar A, Birmingham-Babauta SA, Tomita TM. Relationships and predictive capabilities of jump assessments 
to soccer-specific field test performance in Division I collegiate players. Sports, 2016; 4: doi:10.3390/sports4040056

Maggioni MA, Bonato M, Stahn A, La Torre A, Agnello L, Vernillo G, Castagna C, Merati G. Effects of ball drills and repeated-sprint-ability training in basketball players. Int J Sports Physiol Perform, in press; doi:10.1123/ijspp.2018-0433

McFarland I, Dawes JJ, Elder CL, Lockie RG. Relationship of two vertical jumping tests to sprint and change of direction speed among male and female collegiate soccer players. Sports, 2016; 4: doi:10.3390/sports4010011

Mikolajec K, Waskiewicz, Z, Maszczyk A, Bacik B, Kurek P, Zajac, A. Effects of stretching and strength exercises on speed and power abilities in male basketball players. Isokinet Exerc Sci, 2012; 20(1): 61-69

Padulo J, Laffaye G, Haddad M, Chaouachi A, Attene G, Migliaccio GM, Chamari K, Pizzolato F. Repeated sprint ability in young basketball players: one vs. two changes of direction (Part 1). J Sports Sci, 2015; 33: $1480-1492$

Payne KA, Berg K, Latin RW. Ankle injuries and ankle strength, flexibility, and proprioception in college basketball players. J Athl Training, 1997; 32: 221-225

Pehar M, Sekulic D, Sisic N, Spasic M, Uljevic O, Krolo A, Milanovic Z, Sattler T. Evaluation of different jumping tests in defining position-specific and performance-level differences in high level basketball players. Biol Sport, 2017; 34: 263-272

Pfile KR, Gribble PA, Buskirk GE, Meserth SM, Pietrosimone BG. Sustained improvements in dynamic balance and landing mechanics after a 6-week neuromuscular training program in college women's basketball players. J Sport Rehabil, 2016; 25: 233-240

Shalfawi SA, Sabbah A, Kailani G, Tonnessen E, Enoksen E. The relationship between running speed and measures of vertical jump in professional basketball players: a field-test approach. J Strength Cond Res, 2011; 25: 3088-3092

Spiteri T, Newton RU, Binetti M, Hart NH, Sheppard JM, Nimphius S. Mechanical determinants of faster change of direction and agility performance in female basketball athletes. J Strength Cond Res, 2015; 29: $2205-2214$

Wisløff U, Castagna C, Helgerud J, Jones R, Hoff J. Strong correlation of maximal squat strength with sprint performance and vertical jump height in elite soccer players. Br J Sports Med, 2004; 38: 285-288

Young W, McLean B, Ardagna J. Relationship between strength qualities and sprinting performance. J Sports Med Phys Fitness, 1995; 35: 13-19

\section{Corresponding author:}

\section{Dr. Robert Lockie}

California State University, Fullerton

Department of Kinesiology

800 N State College Blvd

Fullerton, CA 92831, USA

Phone (international): +1 657-278-4971

Email: rlockie @fullerton.edu 